Est Ag 50 (2015) 197-220

\title{
El Depositum Fidei en la Iglesia de Esmirna (siglos I-IV)
}

\author{
MAURICIO SAAVEDRA, OSA
}

RESUMEN: Los diversos modelos de símbolos de fe neotestamentarios están presentes en la documentación de la Iglesia de Esmirna y son usados en contextos catequéticos, bautismales, litúrgicos, así como en contextos de persecución y controversia frente a paganos, judíos y herejes. Es particularmente importante la profesión de fe de los presbíteros de Esmirna en que se proclama la unicidad de Dios frente a las ideas gnósticas y marcionitas, a la vez que se hace la distinción entre Dios y Cristo frente a la coyuntura suscitada por Noeto y la confesión de la verdadera pasión, muerte y resurrección del Señor frente al docetismo. Esta confesión de fe, de carácter normativo, pudo ser un símbolo de fe bautismal usado por la comunidad a finales del siglo II e inicios del siglo III.

PAlABRAS Clave: Iglesia de Esmirna, símbolos de fe, gnósticos, marcionitas.

ABSTRACT: The various types of symbols present in the NT faith recur in the documents of the Church of Smyrna; they are employed in catechetical, baptismal, and liturgical contexts, as well as in the context of persecutions and controversies against pagans, Jews and heretics. The profession of faith of the elders of Smyrna is particularly important; in it is proclaimed the oneness of God against the Gnostic and Marcionite ideas, while the distinction between God and Christ is raised against Noetus, and the confession of the true passion, death and resurrection is directed against Docetism. This normative confession of faith might have been a symbol of baptismal faith used by the community in the late second and early third centuries.

Keywords: The Church of Smyrna, Creeds, Gnostics, Marcionites

La Iglesia de Esmirna es una comunidad fundamental para la comprensión del cristianismo primitivo y el desarrollo del depositum fidei. En efecto, ella es destinataria de una de las cartas del Apocalipsis y se precia 
de tener un origen apostólico ligado a la figura del apóstol Juan. Entre los grandes personajes relacionados con esta Iglesia están el obispo mártir Policarpo, Ignacio de Antioquía quien visitó esa comunidad en su camino hacia Roma e Ireneo de Lyon quien habitó en sus primeros años de vida en Esmirna. Esta comunidad tuvo estrechas relaciones con otras comunidades del mundo cristiano en Asia, Grecia, Galia y Roma, fue defensora de la pascua cuartodecimana, realizó una interesante copia y producción textual y jugó un importante papel en la controversias teológicas con el docetismo, el marcionismo, la Nueva Profecía y la doctrina patripasiana de Noeto, entre otros muchos particulares.

En el contexto de la formación de la ortodoxia frente a diversos grupos y tendencias, analizaremos en este artículo el paulatino proceso en el que el contenido de la fe de la Iglesia de Esmirna viene precisado y se hace vinculante a partir del desarrollo de la lex orandi y la lex credendi.

En el ámbito en el que la aversión de la Escritura judía se manifiesta en los grupos gnósticos y el marcionismo, y en que por otra parte, los cristianos judaizantes, de proveniencia judía o pagana, subrayan la importancia del Antiguo Testamento para la comprensión de la nueva fe, es que surge también paulatinamente la percepción de que una completa libertad de pensamiento termina por perjudicar la concordia y la unidad de la comunidad, y por tanto, se advierte gradualmente la necesidad de precisar el contenido de la fe y de hacerlo vinculante ${ }^{1}$.

Fruto de esta necesidad y en el contexto de finales del siglo II es acuñada por Ireneo ${ }^{2}$, y por otros autores como Tertuliano ${ }^{3}$ y Clemente de

${ }^{1}$ Cf. M. Simonetti, Il Vangelo e la storia, 52.

${ }^{2}$ La regla de la verdad es para Ireneo la doctrina predicada por los apóstoles, transmitida por la sucesión y enseñada en las iglesias. Es el criterio que permite distinguir las falsas interpretaciones de la Escritura de aquellas verdaderas. La regla de la verdad es una enseñanza transmitida tanto por las Escrituas como por la Tradición. Ireneo emplea la

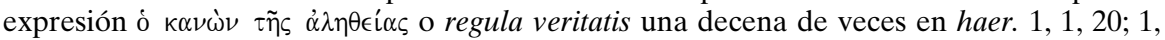
$15 ; 2,40,1 ; 3,2,1 ; 3,11,1 ; 3,12,7 ; 3,15,1 ; 4,57,4$. Ireneo emplea el término regula fidei en el mismo sentido de regula veritatis, cf. Iren., dem. 3 (SCh 406, 86).

3 Para Tertuliano la regula es norma, principio, prescripción. También las escuelas filosóficas y las herejías tienen sus reglas, cf. Tert., praescr. 42, 7 (SCh 46, 149). Para Tertuliano la palabra regula tiene dos sentidos. En un primer sentido regula como el tenor fijo, el contenido determinado de la doctrina. En un segundo sentido regula como la doctrina misma, también muchas veces identificándola con la fe y la verdad o uniendo estos dos conceptos en genitivo a la palabra regula, es decir: regula fidei o regula veritatis. Sin embargo, Tertuliano distingue entre la regla de la fe, que comporta dogmas inmutables como la Trinidad, la Encarnación, la Iglesia, y la regla de la disciplina que comprende usos y prácticas eclesiásticas que pueden cambiar. Como Ireneo, Tertuliano también afirma que la regla de la verdad es una enseñanza a la vez trasmitida por las Escrituras y la Tradición. Sin embargo, a diferencia de las herejías, que son posteriores, la regla de la verdad 
Alejandría 4 la expresión técnica regula fidei o regula veritatis la cual no es un concepto abstracto sino fórmulas efectivas en cada circunstancia en la cual la fe cristiana ortodoxa sienta una posición vinculante sobre conceptos fundamentales considerados inmutables para tomar distancia de concretas desviaciones doctrinales como el gnosticismo, el marcionismo, el docetismo, el patripasianismo, etc. La regula fidei no es sólo un formulario o un símbolo de fe aunque muchas veces el símbolo de fe es la expresión principal de la regula fidei pues esta hace parte de la enseñanza viva de la Iglesia y expresa el matiz fijo e inmutable de la misma. La regula fidei es la parte principal e invariable del depósito apostólico cuya negación o alteración constituye la herejías.

Nos proponemos entonces tratar aquí el proceso de consolidación del deposito de la fe en la Iglesia de Esmirna, no sin antes detenernos brevemente en la construcción del deposito de la fe en el Nuevo Testamento.

\section{La confesiones de fe en el Nuevo Testamento}

La idea de que los miembros de la comunidad de seguidores de Jesús deben permanecer fieles al mensaje transmitido por Él y pos sus apóstoles, mantener su autenticidad y no admitir mezcla alguna con doctrinas extrañas ya aparece en los escritos del Nuevo Testamento. En estos escritos está ya presente la referencia a los principales artículos de la fe, al $\kappa \dot{\eta} \quad \rho u \gamma \mu \alpha$. Se trata del Evangelio y de su mensaje, de su autoridad única en el orden de la fe y de las condiciones de fidelidad a su verdad, pues existe ya en fase de consolidación una conciencia viva de que la fe cristiana supone una normatividad, una regla, unos artículos de fe que deben ser proclamados y defendidos.

está desde el inicio del evangelio. Tertuliano usa la expresión regula veritatis cuatro veces, cf. Tert., apol. 47; adv. Hermog. 1; pud. 8, 12; adv. Marc. 5, 20. La expresión regula fidei la usa ocho veces, cf. Tert., praescr. 12, 5; 13, 1; 26, 9; adv. Marc. 4, 2; virg. vel. 1; Adv. Prax. 3; monog. 2; pud. 19, 3; ieiun.1.

4 En Clemente de Alejandría la palabra $\kappa \alpha \nu$ $\omega$ v es frecuentemente utilizada en el sentido de criterio o regla de conducta y como sinónimo de la expression técnica regula

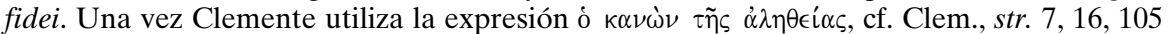
(SCh 428, 316). Clemente comparte en líneas generales la concepción de Ireneo sobre la regula fidei. Para un estudio profundo sobre la regula fidei en estos autores y otros más como Novaciano y Orígenes, cf. D. Van de Eyne, Les Normes de l'Enseigement Chrétien, Paris 1933, 281-313; P. Grech, The Regula fidei as Hermeneutical Principle Yesterday and Today, en Il messaggio biblico e la sua interpretazione, 145-161.

${ }^{5}$ Cf. D. Van de Eyne, Les Normes de l'Enseigment Chrétien, 312-313. 
En la polémica emprendida por Pablo contra los gálatas judaizantes, este proclama el anatema contra todo el que anuncie un evangelio diferente del que él mismo ha proclamado ${ }^{6}$. La decisión colegial que aparece en los Hechos de los Apóstoles en relación con la negativa a imponer las observancias judías a los cristianos procedentes del paganismo señala una normatividad de carácter práctico pero también teórico acerca de la justificación7. La preocupación por la "ortodoxia", en el sentido etimológico de la palabra, o sea por el mantenimiento de la autenticidad de la fe frente a las desviaciones que la acechaban, se expresa ampliamente en las llamadas cartas Pastorales ${ }^{8}$. La 2 Pedro advierte contra "pseudo-profetas" y "pseudo-maestros" capaces de introducir en el pueblo doctrinas perniciosas9. Se condenan ciertas tendencias y grupos que ponen en discusión la doctrina de la Iglesia o que no se inscriben en la comunión de

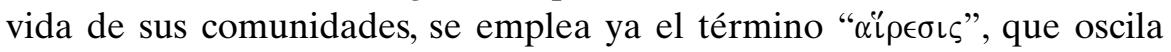
entre el sentido de "escuela", de "secta" o de "facción", con un significado cada vez más peyorativo en el ámbito cristiano10.

Por otra parte, la referencia al Evangelio y la permanencia fiel al mensaje que este transmite se resume en la palabra "tradición ( $\alpha \alpha \rho \alpha ́ \delta o \sigma \iota \varsigma)$ ". Ya en la 1 Tesalonicenses Pablo "dio instrucciones" de parte del Señor Jesús, y ellos recibieron su enseñanza11. Pablo entiende bajo el concepto de tradición el mensaje de la fe ${ }^{12}$, las reglas relativas a la vida interna de las comunidades ${ }^{13}$ y el ideal del comportamiento cristiano ${ }^{14}$. El origen de esta tradición es el Señor mismo ${ }^{15}$. En Lucas y en Juan la idea de tradición es expresada a través de la palabra "testimonio"16. El origen último de esta trasmisión es Dios mismo, que envió a su Hijo único y "lo entregó a la muerte por todos nosotros"17, esto es, lo trasmitión 18 .

${ }^{6}$ Cf. Gal. $1,8-9$.

${ }^{7}$ Cf. Act. 15.

8 Cf. 1 Tim. 6, 3-6; 2 Tim. 4, 1-4; Tit. 3, 10-11.

${ }^{9}$ Cf. 2 Ptr. 2,1.

10 Cf. 1 Cor. 11, 19; Gal. 5, 20; 2 Ptr. 2, 1; Tit. 3, 10.

11 Cf. 1 Thess. 4, 1-2.

12 Cf. 1 Cor. $15,1-5$

13 Cf. 1 Cor. 11, 2; 2 Thess. 2, 15; 3, 6

14 Cf. Phil. $4,9$.

15 Cf. 1 Cor. 11,23

16 Cf. Lc. 24, 48-49; Act. 1, 8.22; 2, 32; Io. 15, 17; 19, 35.

17 Cf. Rom., 8, 32

18 Encontramos esta misma idea de tradición en Ignacio en Eph. 6, 1: “A todo el que envía el Padre de familia a su propia administración, no de otra manera hemos de recibirle que al mismo que le envía", o también en Magn. 7, 1: "A la manera que el Señor 
Este depósito de fe recibido a través de la tradición viene expresado muchas veces por medio de símbolos de fe primitivos que aparecen por todo el Nuevo Testamento y que simplificando un tanto las cosas, siguiendo el aporte de P. Grech y J. Kelly ${ }^{19}$, se presentan principalmente bajo tres modelos.

El primer modelo es a través de una fórmula solo referida a Cristo. Jesús es Señor (Rom. 10, 9; Phil. 2, 11; 1 Cor. 12, 3), Jesús es el Cristo (Act. 18, 5. 28; 1 Io. 2, 22; Act. 8, 36-38), Jesús es el Hijo de Dios (Act. 8, 36-38). También esta confesión a Jesús puede estar constituida por una forma narrativa más o menos desarrollada. Se cuenta en ello el acontecimiento Jesús insistiendo en su misterio de muerte y resurrección, son las proclamaciones, el кń 2, 14-39; 3, 12-26; 4, 9-12; 5, 29-32; 10, 34-43) o por Pablo en sus cartas, por ejemplo: "Yo os trasmití, en primer lugar, lo que a mi vez recibí: que Cristo murió por nuestros pecados según las Escrituras; que fue sepultado y resucitó al tercer día según las Escrituras; que se apareció a Pedro y luego a los doce", (1 Cor. 15, 3-5; Phil. 2, 6-11; Act. 13, 16-41). Estas fórmulas del primer anuncio se ponen en labios de quienes enseñan, mientras que las fórmulas breves expresan más bien la respuesta del creyente. Estas formulaciones kerigmáticas ejercen sobretodo una función doctrinal, sirven de base para la catequesis. Aquella breves expresan más formalmente la función confesante ya que su tenor literario resulta especialmente adaptado a ello.

En un segundo momento existe también la fórmula binaria que comprende la mención del Padre y del Hijo (o de Cristo), esta supone la denominación intencional de los nombres de Dios Padre y de Cristo. Con cada uno de ellos se relaciona una intervención propia en la historia de la salvación. Lo vemos en esta fórmula típica: "Para nosotros no hay más

nada hizo sin contar con su Padre, hecho como estaba una sola cosa con Él -nada digo, ni por sí mismo ni por sus apóstoles- así vosotros nada hagáis vosotros sin contar con vuestro obispo y los ancianos". El término "tradición" sigue siendo de todas formas raro en los Padres Apostólicos. Clemente recomienda a los Corintios que se conformen "a la gloriosa y venerada regla de nuestra tradición”, cf. 1 Clem., 7, 2 (SCh 167, 110; E. Prinzivalli - M. Simonetti, Seguendo Gesù, 188). Clemente es perfectamente consciente de la misma y del origen de este movimiento de envío y transmisión: "Los apóstoles nos predicaron el Evangelio de parte del Señor Jesucristo; Jesucristo fue enviado de Dios. En resumen, Cristo de parte de Dios, y los apóstoles de parte de Cristo: una y otra cosa, por ende, sucedieron ordenadamente por voluntad de Dios", cf. 1 Clem., 42, 1-2 (SCh 167, 168; E. Prinzivalli M. Simonetti, Seguendo Gesù, 238-240).

19 Cf. P. Grech, Le confessioni di fede in Giovanni, en Il messaggio biblico e la sua interpretazione, 333-342. J. Kelly, I simboli di fede della chiesa antica. Nascita, evoluzione, uso del Credo, Bologna 2009, 31-55. 
que un Dios: el Padre, de quien proceden todas las cosas y para quien nosotros existimos; y un Señor, Jesucristo, por quien han sido creadas todas las cosas y por quien también nosotros existimos", (1 Cor. 8, 6; cf. 1 Tim. 2, 5-6; 6, 13).

Por último, existe también una fórmula ternaria que enumera al Padre, al Hijo y al Espíritu. Esta la encontramos en los saludos de Pablo en sus cartas $(2$ Cor. 13,13), o en referencia a las diversas realidades de la vida de la Iglesia en la unidad de un solo misterio, por ejemplo: "Hay diversidad de carismas, pero el Espíritu es el mismo. Hay diversidad de ministerios, pero el Señor es el mismo. Hay diversidad de actividades, pero uno mismo es el Dios que activa todas las cosas en todos", (1 Cor. 12, 4-6; cf. Eph. 4, 4). Otro origen de esta fórmula ternaria parece ser de procedencia litúrgica o bautismal y es con la que se cierra el evangelio de Mateo: "Poneos, pues, en camino haced discípulos a todos los pueblos y bautizadlos para consagrarlos al Padre, al Hijo y al Espíritu Santo, enseñándoles a poner por obra todo lo que les he mandado" $(M t .28,19-20)$. Este texto aunque no pertenece a la tradición apostólica común, ejercerá una influencia decisiva en el desarrollo de las fórmulas bautismales ${ }^{20}$.

A continuación veremos como estos tres modelos de símbolos de fe están presentes en los escritos en torno a la Iglesia de Esmirna.

\section{La lex orandi en la Iglesia de Esmirna del siglo II}

Haciendo un estudio de las fórmulas de fe que aparecen en las fuentes sobre la comunidad de Esmirna encontramos que Ignacio, Policarpo y el autor del Martirio de Policarpo hacen un uso semejante de los modelos de confesiones de fe presentes en el Nuevo Testamento.

Respecto a la fórmula confesional simple, "Jesús es Señor", encontramos que esta adquiere un significado relevante frente a la proclamación "César es Señor" la cual tenía un valor religioso en el mundo pagano. El testimonio que se da de la fe durante la persecución constituirá el contexto privilegiado para el sentido de esta fórmula cristiana, como queda claro en $M$. Polyc., 8, 2 cuando aquellos que estaban alrededor del obispo de Esmirna lo intentaban persuadir para que renegase de su fe: "Le salieron al encuentro el jefe de la Policía, Herodes, y su padre, Nice-

20 En la opinión de O. Cullman conviene aplicarle a estas últimas el nombre de fórmulas ternarias más que trinitarias porque el término "trinidad" no aparece en el Nuevo Testamento, cf. O. Cullmann, La foi et le culte de l'Eglise primitive, Neuchâtel 1963, 91. 
tas, que tras pasarlo a su carruaje y sentarlo, trataban de persuadirlo diciéndole: "Qué malo hay en decir: "César es el Señor", en sacrificar y en todo lo demás y salvarse así?"21.

Esta fórmula simple que hace alusión solo a Cristo, está también firmemente atestiguada en las cartas de Ignacio, aunque ciertamente más desarrollada en sus elementos:

Por tanto, haceos los sordos cuando alguien os hable a no ser de Jesucristo, el de la descendencia de David, el hijo de María, que nació verdaderamente, que comió y bebió, que fue verdaderamente perseguido en tiempo de Poncio Pilato, que fue crucificado y murió verdaderamente a la vista de los seres celestes, terrestres e infernales. Él resucitó verdaderamente de los muertos, habiendo sido resucitado por su mismo Padre, y, a semejanza suya, a los que hemos creído en Él también su Padre nos resucitará en Jesucristo, fuera del cual no tenemos vida verdadera 22 .

La fórmula ignaciana está marcada por el cuño de la controversia, al mismo tiempo que repite el mensaje tradicional. El adverbio "verdaderamente" repetido cuatro veces, hace manifiesta la probable intención de oponerse a los herejes "docetas" que se niegan a creer en la verdadera humanidad de Jesús. La confesión de fe se convierte de este modo en una "regla de fe" que distingue a los verdaderos cristianos de aquellos que no lo son. Esta insistencia del adverbio, exigida por las circunstancias, no le sobrevivirá.

Las mismas características de la anterior y el mismo sabor antidoceta nos queda en este otro apartado que se encuentra en la carta de Ignacio a la comunidad de Esmirna:

Glorifico a Jesucristo, Dios, que os ha concedido tal sabiduría. Pues he sabido que habéis alcanzado la perfección en la fe inconmovible de manera que estáis clavados en la carne y en el espíritu a la cruz del Señor Jesucristo, sólidamente establecidos en el amor por la sangre de Cristo y

${ }^{21}$ M. Polyc. 8, 2, cf. Rom. 10, 9; Phil. 2, 11; 1 Cor. 12, 3. El cristiano no podía admitir esa fórmula en la que veían la negación del único señorío de Cristo, cf. 1 Cor. 8, 5-6. Cf. $M$. Polyc., 17, 3: "A Él lo adoramos como Hijo de Dios".

22 Ign., Trall. 9, 1-2: cf. 1 Cor. 15, 3-5; Phil. 2, 6-11; Act. 13, 16-41. Este texto es importante por varios aspectos. Reproduce, de forma bien acuñada, la secuencia kerigmática heredada de la tradición neotestamentaria. Pero introduce algunas novedades notables. Menciona el nacimiento virginal de Jesús, un dato que siempre estuvo ausente en los kerigmas del Nuevo Testamento, pero que pertenece en adelante a todos los credos cristológicos. Recoge igualmente la mención que se hará clásica: "bajo Poncio Pilato", indicación ya presente en Act. 3, 13 y 1 Tim. 6, 13. Cf. Ign., Eph. 18, 2. 
repletos de certeza en nuestro Señor, que es verdaderamente de la estirpe de David, según la carne, hijo de Dios por la voluntad y el poder de Dios, nacido verdaderamente de una virgen, bautizado por Juan para que toda justicia fuese cumplida por Él, crucificado verdaderamente en la carne por nosotros bajo el poder de Poncio Pilato y del tetrarca Herodes -nosotros existimos gracias a su fruto, gracias a su bienaventurada pasión- para levantar un signo por los siglos mediante su resurrección para sus santos y fieles, ya sean judíos, ya sean paganos, en el único cuerpo de la Iglesia ${ }^{23}$.

Encontramos también en Polyc., ep. 7, 1 una fórmula que, aunque breve, engloba la confesión de Jesús dentro del misterio de la encarnación: "Todo el que no confiese que Jesucristo ha venido en carne, es un anticristo" 24 .

Respecto a la fórmula confesional binaria, donde se confiesa al Padre y al Hijo, también hallamos ejemplos en Ignacio y en Policarpo. Ignacio en Magn. 18, 2 dice: "Los profetas también fueron perseguidos al estar inspirados por la gracia de Jesucristo, para que los desobedientes tuvieran la certeza de que existe un único Dios, el cual se ha manifestado por medio de su Hijo Jesucristo, que es su Palabra salida del Silencio"25.

Policarpo en ep. 12, 2 afirma:

Que el Dios y Padre de nuestro Señor Jesucristo y el mismo Pontífice Eterno ${ }^{26}$, Hijo de Dios, Jesucristo, os edifique en la fe, en la verdad, en toda mansedumbre, sin ira, en paciencia, en longanimidad, constancia y castidad; y os de la herencia y la parte entre sus santos, y a nosotros con vosotros y con todos los que están bajo el cielo y han de creer en Jesucristo, nuestro Señor, y en su Padre que lo resucitó de entre los muertos.

También en 2, 1 Policarpo nos trasmite una fórmula breve que es binaria al tiempo de kerigmática: "Creyendo al que resucitó a nuestro Señor Jesucristo de entre los muertos y le dio gloria".

Las fórmulas ternarias, por su parte, abren sin embargo a un porvenir más duradero, Ignacio ya nos da un ejemplo: "Esforzaos en permanecer firmes en las enseñanzas del Señor y de los apóstoles para que todo

23 Ign., Smyrn., 1, 1-2: cf. 1 Cor. 15, 3-5; Phil. 2, 6-11; Act. 13, 16-41.

24 Polyc., ep. 7, 1. Esta fórmula tiene la hechura breve y bien acuñada del primer modelo, pero lo que proclama de Jesús no es un título, sino el acontecimiento global de su encarnación. La misma fórmula "Jesucristo venido en la carne" la encontramos en las cartas juánicas: cf. 1 Io. 4, 2; 2 Io. 7.

25 Esta expresión denota ciertos contactos con la gnosis: cf. J. Ayán Calvo, 133.

26 Cf. 1 Clem. 36, 1; 61, 3 (SCh 167, 158; 200; E. Prinzivalli - M. Simonetti, Seguendo Gesù, 232. 268); Ign., Philad. 9, 1; M. Polyc. 14. 
lo que hagáis sea próspero en la carne y en el espíritu, en la fe y en el amor, en el Hijo, en el Padre y en el Espíritu"27.

Por otra parte, Policarpo tiene en mente, sin duda, la enseñanza tradicional, compacta en su estructura fundamental aunque es plástico en la manera de formularla verbalmente. En ep. 2,1 dice "creyendo en Aquél que resucitó a nuestro Señor Jesucristo de entre los muertos y le dio gloria $^{28}$ y un trono a su derecha ${ }^{29}$. A Él fueron sometidos todos los seres celestes y terrestres ${ }^{30}$; a Él le sirve todo aliento; Él vendrá como juez de vivos y de muertos" 31 .

La estructura de esta última confesión se hace en dos cláusulas y el elemento más largo concerniente a la cristología está subordinado al primer elemento que es más breve. Probablemente, se trata de un fragmento que venía enseñado habitualmente a los convertidos de la Iglesia de Esmirna y que Policarpo usa en esta carta ${ }^{32}$.

Sin embargo, la carta $A$ los filipenses, que en cierto modo refleja también la enseñanza de Policarpo a su propia comunidad de Esmirna, tiene más una impostación ética en la cual se recomienda la imitación de Cristo, que una impostación doctrinal, aunque también como hemos visto, introduce explícitas professiones fidei.

Para Policarpo la caridad y la fe en Cristo se funden. Quien cree practica la caridad y quien practica la caridad demuestra su amor "por Dios, por Cristo y por el prójimo"33. Policarpo al exhortar el vivir cumpliendo "los mandamientos de la justicia" 34 reitera los principios de la fe recapitulándolos en Cristo. La fe en Cristo es la razón y el fin de un comportamiento virtuoso:

Así pues, perseveremos sin cesar en nuestra esperanza y en las arras de nuestra justicia, que son Jesucristo, el cual sobre la cruz levantó nuestros

27 Ign., Magn. 13, 1. Es interesante que algunos renglones después Ignacio vuelve sobre la misma fórmula pero haciendo la invitación a someterse al obispo y también los unos a los otros "como Jesucristo al Padre, según la carne, y los apóstoles a Cristo, al Padre y al Espíritu, para que la unidad sea carnal y espiritual”, Ign., Magn. 13, 1.

28 Cf. 1 Ptr. 1, 21.

29 Cf. 1 Clem. 65, 2 (SCh 167, 204; E. Prinzivalli - M. Simonetti, Seguendo Gesù, 274).

30 Cf. 1 Ptr. 3, 22.

31 Cf. 1 Ptr. 4,5 .

32 Merece la pena colocar atención en el hecho de que se trata de varias citas sacadas probablemente de 1 Pedro o 1 Clemente, lo que manifiesta el modo en que iba siendo construido el cuerpo de la tradición catequética, cf. J. Kelly, I simboli di fede della chiesa antica, 109.

33 Cf. Polyc., ep. 3, 2.

${ }^{34}$ Cf. Polyc., ep. 3, 3. 
pecados en su propio cuerpo, a pesar de que no cometió pecado ni se encontró engaño en su boca. Pero todo lo soportó por nosotros para que viviéramos en Él. Así pues, seamos imitadores de su paciencia y, si padecemos por su nombre, glorifiquémosle. Pues ese ejemplo, nos lo puso en su propia persona, y nosotros le hemos creído ${ }^{35}$.

El M. Polyc., 14, 1 nos trasmite una oración puesta en boca del obispo de Esmirna inmediatamente antes de su martirio ${ }^{36:}$

Señor, Dios todopoderoso, Padre de tu amado y bendito siervo Jesucristo, por el que te hemos conocido, Dios de los ángeles, de las potencias, de toda la creación y de todo el pueblo de los justos, que viven en tu presencia. Te bendigo porque me has juzgado digno de este día y de esta hora, de tomar parte en el número de tus mártires, en el cáliz de tu Cristo, para resurrección de la vida eterna en el alma y en cuerpo, en la incorruptibilidad del Espíritu Santo. Que hoy sea yo recibido con ellos en tu presencia, en sacrificio generoso y grato, tal como Tú, el Dios verdadero que no engaña, lo has preparado de antemano, lo anunciaste y lo has cumplido. Por ello y por encima de todas las cosas te alabo, te bendigo, te glorifico, por medio de Jesucristo, Sumo Sacerdote eterno y celeste, tu amado siervo, por el cual la gloria sea dada a Ti junto a Él y al Espíritu Santo, ahora y en los siglos venideros. Amén.

En esta oración podemos encontrar una fórmula ternaria en donde el Dios Padre todopoderoso encabeza la fórmula, Jesucristo es confesado como Sumo Sacerdote eterno y celeste, amado siervo del Padre y el Espíritu Santo es calificado como incorruptible y sujeto de la gloria junto al Padre y al Hijo. Es relevante que califica a Dios Padre como Dios de toda la creación quizá en claro alusión antimarcionista, de igual forma confiesa la resurrección de la vida eterna en alma y cuerpo.

Esta oración muy probablemente fue confeccionada por el mismo redactor del Martirio de Policarpo. Eventualmente, este oración pudo haber tenido un uso litúrgico posterior cuando se recordaba la memoria de Policarpo ${ }^{37}$. No se puede excluir que durante la celebración de esta

35 Cf. Polyc., ep. $8,1$.

36 Para un estudio extenso acerca de la oración de Policarpo, cf. D. Tripp, The Prayer of St Polycarp: its setting, its roles and its text, en Ephemerides Liturgicae 104 (1990), 97-132.

37 M. Polyc., 18, 3. 
memoria fueran leídos al menos extractos de esta carta, de los cuales muy seguramente este oración pudo tener un lugar especial ${ }^{38}$.

\section{La profesión de fe de los presbíteros de Esmirna}

A finales del siglo II encontramos en Esmirna la difícil situación comunitaria y doctrinal generada por el presbítero cismático Noeto ${ }^{39}$. De aquella confrontación nos ha llegado una profesión de fe que merece especial atención. A diferencia de las otras confesiones de fe que hemos encontrado en Ignacio, en Policarpo y en el Martirio de Policarpo, nos encontramos ante el único texto confesional de carácter "oficial" que encontramos en nuestra documentación sobre la Iglesia de Esmirna. Este texto es "oficial" en el sentido que es el único texto verdaderamente normativo y vinculante que conocemos.

Aunque no conocemos ad litteram la profesión de fe de Noeto, sí ha llegado a nosotros la profesión de fe de los presbíteros de Esmirna: "También nosotros conocemos verdaderamente $(\dot{\alpha} \lambda \eta \theta \tilde{\omega} \varsigma)$ un solo Dios, conocemos a Cristo, conocemos al Hijo que ha padecido como ha padecido, murió como murió, y resucitó al tercer día, está a la derecha del Padre y viene a juzgar a los vivos y a los muertos".

Con la precisión " $\alpha \lambda \eta \theta \tilde{\omega} \varsigma$ " los presbíteros de Esmirna distinguen su fe de aquella de Noeto, la cual consideraron errónea. Así la profesión de fe de los presbíteros es a su vez una regula fidei, es decir, un conjunto de conceptos doctrinales considerados inmutables finalizados a sentar una posición clara y unívoca frente a determinadas tendencias doctrinales consideradas no ortodoxas ${ }^{40}$.

38 No poseemos más información sobre el uso litúrgico de las actas martiriales en el Asia en los primeros siglos. Sabemos, por otra parte, que en África cuando celebraban la memoria de un mártir se solían leer las actas de este durante la ceremonia. En efecto, personajes como Agustín, en su sermones de estas fiestas de mártires, no recuerda todos los detalles del relato martirial, solamente alude a algunos episodios, puesto que ya previamente en la misma ceremonia las actas habían sido leídas. Este uso africano se diferencia de los panegíricos griegos de Basilio, Gregorio de Nisa, Juan Crisóstomo, etc., que recuerdan detalladamente todos los particulares del martirio, justamente porque la lectura de las actas muy seguramente no estaba prevista. Por su parte, en Roma era prohibida la lectura de las actas de mártires durante la liturgia ya que muchas de estas contenían muchos hechos ficticios, cf. V. Saxer., Morts, Martyrs, Reliques, en Afrique Chrétienne aux Premiers Siècles, Roma 1980, 200-208.

39 Toda la problemática en torno a Noeto, cf. Edición de M. Simonetti, Ippolito, Contro Noeto, Biblioteca Patrística, Bologna 2000.

40 Cf. D. Van de Eyne, Les Normes de l'Enseigement Chrétien, 312. 
La profesión de fe de los presbíteros sintetiza en gran medida los conceptos fundamentales que la Iglesia de Esmirna a finales del siglo II subraya frente a las diversas desviaciones doctrinales.

La confesión de fe en "un solo Dios", aparte de la impostación genéricamente antipagana, es seguramente una declaración de los presbíteros de Esmirna que se preocupan por proclamar esta verdad frente al gnosticismo y al marcionismo que compartían el fundamental concepto de la distinción entre el dios inferior de los judíos defensor de la justicia y creador del mundo, y el dios sumo y bueno, el dios desconocido que solo Cristo había revelado a quien hubiese creído en él.

A su vez, los presbíteros de Esmirna en su profesión de fe distinguen a Dios de Cristo ("conocemos un solo Dios... conocemos a Cristo"), especificando de este último solamente lo que respecta a la encarnación ${ }^{41}$. Es decir, los presbíteros de Esmirna rechazan de Noeto la afirmación de que Dios Padre se haya encarnado en Cristo.

Por último, el verdadero padecimiento, muerte y resurrección del Señor subraya una vez más la verdadera humanidad de Cristo frente al docetismo ya presente en el ámbito asiático desde finales del siglo I e inicios del siglo II y combatido anteriormente por Ignacio y Policarpo.

Según J. Kelly, los elementos de la profesión de fe de los presbíteros de Esmirna parecen ser parte de un símbolo de fe en uso, quizá bautismal, por parte de la comunidad a finales del siglo II $^{42}$.

A partir de este estudio acerca de las fórmulas confesionales en torno a la comunidad de Esmirna podemos aproximarnos a la realidad qué delatan. Aquellas confesiones de fe que van dirigidas a las comunidades, aunque si bien pueden brotar de ambientes litúrgicos y catequéticos, tienen también la finalidad de mantener a los creyentes firmes en una enseñanza recibida, la cual posiblemente corre el peligro de perderse o de tergiversarse por grupos al externo o al interno de la misma comunidad. Agentes externos a la comunidad como paganos y judíos, tendencias al interno de las comunidades cristianas ya presentes desde el ambiente neotestamentario como cristianos judaizantes y docetas, realidades más o menos difíciles de individuar como el gnosticismo y el marcionismo y reacciones a la doctrina del Logos que aparece a los ojos de muchos como lesiva del tradicional theologoumenon de la unicidad de Dios ${ }^{43}$.

41 Cf. M. Simonetti, Contro Noeto, 199. Hipólito siguiendo la teología de Justino e Ireneo, en su homilía Contra Noeto no solo hará alusión a la encarnación del Logos, sino a su preexistencia.

${ }^{42}$ Cf. J. Kelly, I simboli di fede della chiesa antica, 82.

${ }^{43}$ Cf. M. Simonetti, Contro Noeto, 45. 
Frente al peligro de que esta enseñanza se pierda o se tergiverse por estos agentes, las confesiones de fe manifiestan una precisa regula fidei $\mathrm{o}$ regula veritatis finalizada a identificar la fe cristiana vinculante en cada circunstancia particular.

La confesión de fe de los presbíteros de Esmirna es un texto que, aunque breve, señala, como hemos visto, claros conceptos vinculantes para la Iglesia de Esmirna y es un punto de llegada seguro y fundamental del desarrollo doctrinal de esta comunidad.

\section{La lex credendi en la Iglesia de Esmirna}

Tratando de identificar aún más esta correlación de fuerzas al interno de la comunidad de Esmirna examinaremos la concepción de ortodoxia y herejía que pueden manejar nuestros autores y la formación de la lex credendi de la comunidad.

En lo que respecta a la visión que tenía Ignacio acerca de la defensa de una fe ortodoxa, sabemos que su aproximación, sobretodo, en las cartas que dirige a la Iglesia de Esmirna y a Policarpo, se hace fundamentalmente a la luz del tema de la unidad eclesial. Es ello lo que le impulsa a combatir la herejía que es la peor de las asechanzas o malas artes ${ }^{44}$ con que Satanás intenta apartar a los creyentes de la nueva vida que Cristo ha hecho posible con su encarnación, pasión y resurrección. Si Cristo había sido ungido para, a su vez, comunicar incorrupción a su Iglesia, el príncipe de este mundo quiere ungir a los creyentes con el pestilente ungüento de su doctrina para, así, tenerlos sometidos y cautivos: "Por esto el Señor tomó ungüento sobre su cabeza para inspirar a la Iglesia incorrupción. No os unjáis la fetidez del príncipe de este mundo para que no os lleve cautivos lejos de la vida que os ha sido propuesta como recompensa" 45 . Ahora bien, el príncipe de este mundo se vale de los herejes que son los que corrompen con su mala doctrina la fe de Dios por la que Cristo fue crucificado: "Así pues, si los que obran estas cosas según la carne -se refiere a los adúlteros- murieron, ¡cuánto más si corrompe en mala doctrina la fe de Dios por la que Cristo fue crucificado!" 46 .

\footnotetext{
44 Cf. Ign., Trall. 8, 1; Philad. 6, 2.

45 Ign., Eph. 17, 1. Cf. A. Orbe, La Unción del Verbo. Estudios Valentinianos III, Roma 1961, 5-13.

46 Cf. Ign., Eph. 16, 2
} 
La herejía para Ignacio, como advierte en la carta $A$ los esmirniotas, va íntimamente ligada a la muerte espiritual y eterna, siendo muy difícil la conversión de los seguidores de tales doctrinas ${ }^{47}$. Así, los herejes ofrecen una doctrina que causa la muerte ${ }^{48}$; son plantas cuyo fruto es mortal ${ }^{49}$; son abogados de la muerte ${ }^{50}$; mueren en sus disputas ${ }^{51}$ e irán al fuego inextinguible ${ }^{52}$. La postura de Ignacio frente a la herejía es clara y contundente. Sin embargo, no es del todo fácil determinar cuáles y cuántos son los grupos heréticos combatidos por Ignacio en todas las cartas que dirige a las iglesias del Asia Menor. No cabe duda, como ya hemos visto por las confesiones de fe, que la polémica antidoceta está presente en casi todas ellas. Por otro lado, en las cartas $A$ los filadelfios y $A$ los magnesios ataca frontalmente la tendencia judaizante a la que seguían ligados algunos miembros de las comunidades. Esto ha conducido a los estudiosos a variadas posturas. Según J. Donahue y J. Rius-Camps, Ignacio combate dos grupos bien diferenciados, docetismo y judaísmo ${ }^{53}, \mathrm{C}$. Trevett incluso adjunta otro grupo de itinerantes carismáticos que están directamente en contra de Ignacio y de la idea que tiene de episcopado ${ }^{54}$. Por el contrario, W. Barnard, P. Prigent y H. Bammel piensan que el obispo de Antioquía se enfrenta a un solo grupo que, fundamentado en categorías judaicas, mantenía así mismo una cristología doceta ${ }^{55}$. Finalmente, E. Norelli identifica sólo el grupo de docetas como adversarios de Igna$\operatorname{cio}^{56}$.

En lo personal, tomando distancia de esquematismos un tanto rígidos y, consiguientemente, poco conciliables con el desenvolvimiento de diversas comunidades cada una con situaciones particulares, estoy de

47 Cf. Ign., Smyrn. 4, 1.

48 Cf. Ign., Smyrn. 6, 2.

${ }^{49}$ Cf. Ign., Trall. 11, 1.

50 Cf. Ign., Smyrn. 5, 1.

51 Cf. Ign., Smyrn. 5, 1.

52 Cf. Ign., Eph. 16, 2.

53 Cf. P. J. Donahue, Jewish Christianity in the Letters of Ignatius of Antioch, en Vigiliae Christianae 32 (1978) 81-93; J. Rius-Camps, The Four Authentic Letters of Ignatius, the Martyr, Roma 1979, 40-51; H. Bammel, Ignatian Problems, en Journal of Theological Studies 33 (1982), 83ss. 194ss.

54 Cf. C. Trevett, A Study of Ignatius Antioch in Syria and Asia, Lewiston 1992,

55 Cf. L. Barnard, Studies in the Apostolic Fathers and their Background, Oxford 1966, 23-27; P. Prigent, L'hérésie asiate et l'Église confessante. De l'Apocalypse à Ignace, en Vigilae Christianae 31 (1977) 1-7.

56 Cf. E. Norelli, Ignazio di Antiochia combatte veramente dei cristiani giudaizzanti?, en Verus Israel. Nuove prospettive sul giudeocristianesimo, ed. G. Filoramo - C. Gianotto,, Brescia 2001, 229ss. 
acuerdo con el estudio de P. Meinhold ${ }^{57}$ y más recientemente el de P. Foster ${ }^{58}$, que propenden por una distinción de los adversarios combatidos por Ignacio que no se pueden reducir a una unidad. M. Simonetti, en efecto, considera que la situación de Magnesia no es necesariamente la misma de Filadelfia o de Esmirna. Por lo demás, teniendo presente el carácter extremista de Ignacio de Antioquía, cabe la posibilidad de que él haya advertido y exasperado situaciones que no eran advertidas tan claramente en la realidad cotidiana de las comunidades asiáticas ${ }^{59}$. Cada carta de Ignacio presenta matices que caracterizan las tensiones doctrinales que se estaban produciendo en cada una de aquellas comunidades. Así pues, la situación podría bosquejarse así: En Éfeso, a pesar de que la comunidad parece unida y compacta entorno a la jerarquía no obstante la presencia de diferentes grupos ${ }^{60}$, existían algunos que criticaban el silencio del obispo quizá frente a la elocuencia de algunos predicadores posiblemente itinerantes. Sin embargo, a estos individuos no es necesario considerarlos gnósticos ${ }^{61}$. En Magnesia existían elementos carismáticos, opuestos a la jerarquía local y con influencias judaizantes. En Trales existía un grupo doceta y quizá una cierta indiferencia sobre el papel del obispo en la comunidad. En Filadelfia ocurría la misma situación que en Magnesia, podría tratarse de grupos de cristianos judaizantes que creaban además una profunda división en la comunidad ${ }^{62}$. Finalmente, en Esmirna la corriente contra la que Ignacio colocó en guardia a la comunidad y a su obispo Policarpo era sobretodo el docetismo, además de una parte de la comunidad que celebraba separadamente la liturgia eucarística y no reconocía la autoridad del obispo ni el ordenamiento jerárquico a tres niveles. Sin embargo, no podemos determinar si los docetas eran aquellos mismos que rechazaban el ordenamiento jerárquico de la comunidad.

57 Cf. P. Meinhold, Studien zu Ignatius von Antiochen, Wiesbaden 1979, 19-36.

58 Cf. P. Foster, The Epistles of Ignatius of Antioch, en The Writings of the Apostolic Fathers, ed. P. Foster, Oxford 2007, 89ss.

59 Cf. E. Prinzivalli - M. Simonetti, Seguendo Gesù, 296.

60 Esta es fundamentalmente la conclusión del libro de Trebilco, cf. P. Trebilco, The Early Christians in Ephesus from Paul to Ignatius, 628-717.

$61 \mathrm{C}$. Trevett ha reconocido también la existencia de esta tendencia, cf. C. Trevett, Prophecy and Anti-Episcopal Activity: A tirad Error Combatted by Ignatius?, en Journal of Ecclesiastical History 34 (1983) 1-18. También identifica estos elementos con las actividades carismáticas que aparecen en la Didaché. Cf. E. Prinzivalli - M. Simonetti, Seguendo Gesù, 321.

$62 \mathrm{C}$. Trevett ha caracterizado las enseñanzas de estos herejes como premontanistas, cf. C. Trevett, Apocalypse, Ignatius, Montanism: Seeking the Seeds, en Vigiilae Christianae 43 (1989) 313-338. 
Así pues, Ignacio solo parece considerar doctrina herética el docetismo, o mejor, una cristología doceta que no se ha de relacionar necesariamente con el gnosticismo ni tampoco con el judaísmo. La polémica con los judaizantes probablemente no es un enfrentamiento con personas externas a la comunidad sino con miembros o grupos integrados en las comunidades eclesiales y relacionados con el vidente del Apocalipsis, miembros o grupos que se oponían a la enseñanza y ética de Pablo. Finalmente, podían existir miembros de la comunidad -no docetas ni judaizantes- que no se sentían cómodos con el desarrollo de las comunidades, pues habituados a un esquema de Iglesia más carismático y menos jerárquico, no aceptaban la visión eclesial de Ignacio, aquellos inconformes serían también gentes procedentes probablemente de círculos juánicos ${ }^{63}$. En este análisis salta el tema del posible choque de tradiciones apostólicas entre la tradición juánica difusa en los cristianos judaizantes y la tradición paulina estimada de modo especial por Ignacio y por Policarpo. En efecto, la común admiración por Pablo pudo haber alimentado la cercanía espiritual entre Ignacio y Policarpo. Sin embargo, esto no quiere decir que para ambos varios elementos de la tradición juánica no fuesen estimables.

Por su parte, Policarpo en su carta $A$ los filipenses tiene un estilo mucho más sereno que el de Ignacio en el combatir las posibles desviaciones doctrinales de esta comunidad. Sin embargo, en 7,1 reprocha a aquellos que no confiesan que Cristo ha venido en la carne y reniegan del testimonio de la cruz ( tarios a abandonar la vanidad de la muchedumbre y sus falsas doctrinas ( $\tau \dot{\alpha} \varsigma \psi \epsilon \in \delta 0-\delta\llcorner\delta \alpha \sigma \kappa \alpha \lambda i \hat{L} \varsigma$ ), a volver a la palabra que fue trasmitida desde el

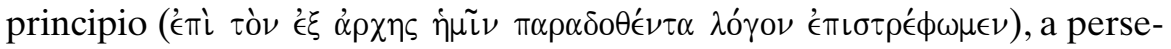
verar en los ayunos y en la oración. Quien no confiesa que Jesucristo ha venido en la carne, quien niega la cruz y quien distorsiona la palabra del Señor para satisfacer sus propias pasiones negando la resurrección y el

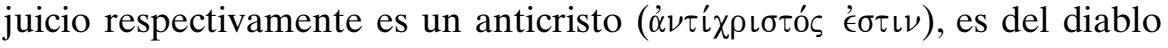

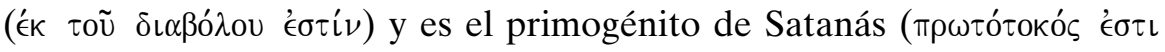

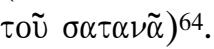

En este pasaje parece claro que Policarpo alude a las corrientes docetistas que al renegar de la encarnación del Verbo, frustran la obra de

63 Cf. C. Trevett, A Study of Ignatius of Antioch in Siria and Asia, Lewiston-Queenston $1992,75-113$ y $147-215$.

64 Cf. Polyc., ep. 7, 1. 
la redención. Así pues, en el contexto de la carta de Policarpo, y también en las de Ignacio, el sustantivo $\alpha \nu \tau i ́ x \rho \iota \sigma \tau o \varsigma$ viene a significar el falso maestro y el falso hermano ${ }^{65}$. El anticristo obra en el presente, corrompe y engaña, provoca la muerte porque mata el espíritu de verdad que está en los hermanos. Por eso el único remedio contra los "anticristos" es el de "volver a la palabra que nos fue transmitida desde el principio" 66.

Por su parte, el Martirio de Policarpo, parece no hacer una mención especial a cuidarse de desviaciones doctrinales y en este sentido no expresa calificativos contra miembros de la comunidad que puedan estar atentando contra la fe recibida. Algunos estudiosos han querido ver en el personaje Quinto procedente de Frigia un elemento explícitamente antimontanista en el relato del Martirio de Policarpo 467. En efecto, a la breve narración del martirio de Germánico sigue el fallido testimonio de Quinto, el cual se había forzado a sí mismo y a otros a arrojarse a las fieras voluntariamente y al final termina cediendo a la pretensión del procónsul abjurando y sacrificando. La actitud de Quinto no es aprobada por el autor del relato puesto que entregarse espontáneamente no es conforme a la enseñanza del evangelio.

Es claro que el episodio de Quinto está colocado en el relato para hacer frente a un cierto fanatismo religioso. Para algunos, el atribuir directamente este relato a una condena del montanismo, por la mención que hace de la proveniencia frigia del personaje y su manifestación de rigorismo, parece una atribución excesiva viendo el conjunto de todo la carta, ya que en el caso de que el "frigio" equivaliera a "montanista" en este relato, sería la única alusión explícita a esta secta en todo el texto. Sin embargo, llama la atención que el texto señala dos veces en la misma frase la condición "frigia" del personaje: "Pero uno que se llamaba Quinto, frigio ( $\Phi \rho u ́ \xi)$, que había venido recientemente de Frigia ( $\left.\tau \tilde{\eta}_{\varsigma} \Phi \rho v \gamma i \alpha \varsigma\right)$, se acobardó al ver las fieras". Así pues, esta aparente redundancia puede señalar efectivamente la identificación de este personaje con un miembro del incipiente movimiento de Montano.

Aún así, el tema fundamental de la narración, como hemos dicho más arriba, es el martirio $\kappa \alpha \tau \tilde{\alpha} \in \cup \alpha \gamma \gamma^{\prime} \epsilon \iota\llcorner o \nu$ y no la confutación de la doctrina de Montano. Por lo demás, el fallido testimonio de Quinto se opone al

65 Cf. Polyc., ep. 6, 3.

66 Cf. Polyc., ep. 7, 2.

${ }^{6}$ Cf. S. Ronchey, Indagine sul martirio di san Policarpo, Roma 1990, passim; cf. G. Buschmann, Martyrium Polycarpi 4 und der Montanismus, en Vigilae Christianae 49 (1995), 105-145. 
valiente testimonio de Germánico y precede el maravilloso testimonio de Policarpo 68 .

Por otra parte, M. Simonetti69 afirma, aunque con cierta cautela, que el movimiento montanista pudo tener diferentes fases de desarrollo. Así pues, en un primer momento, hacia el año 157 según una noticia de Epifanio, pudo comenzar la predicación de Montano y en estos años se vio sólo a un entusiasta con una ascesis muy rígida, pero sin las connotaciones de desviación doctrinal que adquirirá el movimiento posteriormente, probablemente hacia el año 172, fecha en que Eusebio situó su origen. Así pues, Quinto pudo eventualmente ser un montanista contemporáneo de Policarpo, antes de que los seguidores de montano pudiesen ser identificados claramente como una verdadera secta.

Por el contrario, en el M. Pion., 11, 2, alrededor cien años después del martirio de Policarpo, la alusión a los frigios como pertenecientes a un secta claramente identificada es explícita. Entre los cristianos encerrados en la prisión junto a Pionio se encuentran "un presbítero de la Iglesia

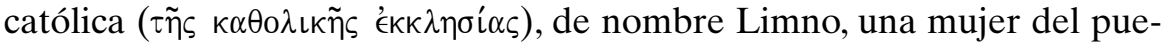

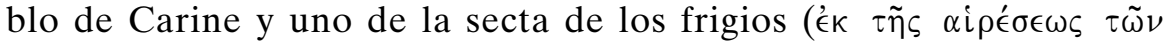
$\Phi \rho u \gamma \tilde{\omega} \nu)$ de nombre Eutiquiano"70. Además, es clara la contraposición entre Iglesia católica y herejía. Los cristianos usaron específicamente el término griego $\kappa \alpha \theta \lambda \iota\llcorner$ เós para significar una diferenciación de los ortodoxos respecto a los herejes ${ }^{71}$. También en el M. Pion., 21, 5 aparece junto a Pionio un tal "Metródoro, presbítero de la secta de los marcionitas ( $\tau \tilde{\eta} \varsigma$

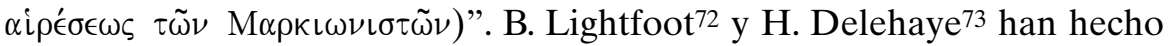
notar que esta mención de herejes martirizados vale como una prueba de la autenticidad, pues de norma la Iglesia se refutaba a reconocer a los herejes como mártires.

El fenómeno de la Nueva Profecía, "los Frigios" o muy posteriormente denominado "montanismo" se comenzó a desarrollar hacia el año

$68 \mathrm{H}$. von Campenhausen ha hecho notar que el capítulo 3 y el capítulo 5 del Martirio de Policarpo podrían unirse sin dificultad, en caso de no existir el capítulo 4 que habla sobre Quinto. Sin embargo, en principio no habría que ver la alusión de Quinto como una interpolación de un antimontanista posterior, cf. H. von Campenhausen, Bearbeitungen und Interpolationen des Polykarps-martyrium, Heildelberg, 1957, 18-21; cf. M. Simonetti, Alcune osservazioni sul martirio di S. Policarpo, en Giornale Italiano di Filologia 4 (1956) 339.

${ }^{69}$ Cf. M. Simonetti, Alcune osservazioni sul martirio di S. Policarpo, 332-340.

${ }^{70}$ M. Pion., 11, 2.

71 Cf. M. Simonetti, Studi Agiografici, 33.

72 Cf. B. Lightfoot, The Apostolic Fathers, 639.

73 Cf. H. Delehaye, Les passions, 29. 
160 por motivos que desconocemos pero que podían depender en gran parte de las esperas milenaristas acaecidas en le región de Frigia y en las regiones limítrofes. En efecto, parece que esta tendencia esperaba el descenso a la tierra de la nueva Jerusalén, signo indicativo de la instauración del milenio en la región de Pepuza. Fue sobretodo la convicción de estos entusiastas de ser directamente inspirados por el Espíritu Santo, más que la espera de la parusía o su riguroso ascetismo, lo que llamó la atención de la jerarquía eclesiástica la cual veía en esta pretensión una amenaza a la estructura jerárquica episcopal de las iglesias locales no solo de la Frigia y del Asia sino de otras partes del imperio como Roma, África y Galia. Así pues, la jerarquía les consideró falsos profetas, les reprocharon sus éxtasis y su actividad profética y carismática. La dificultad de acusar a los montanistas de concretas desviaciones doctrinales exigió de parte de la jerarquía concertar una línea común de comportamiento por la cual se reunieron los obispos locales en varios concilios, especialmente en varios lugares del Asia ${ }^{74}$, y la presión jerárquica fue acompañada de una intensa actividad literaria de tono polémico ${ }^{75}$ y hacia el final del siglo II la crisis era casi ya superada excepto en Frigia y sus alrededores con comunidades montanistas que estuvieron activas hasta el final del siglo IV76.

Dentro de esta actividad literaria antimontanista del siglo III o IV podemos probablemente situar la Vida de Policarpo en el área asiática, y específicamente en Esmirna. Como parte de los aspectos comunes de las biografías intelectuales de la antigüedad, esta vita tiene también un propósito polémico. En efecto, Policarpo es descrito predicando contra la doctrina montanista: "Acerca del Espíritu Santo y don del Paráclito y demás carismas demostraba que no es posible darse fuera de la Iglesia católica, al modo que tampoco un miembro cortado del cuerpo tiene fuerza alguna"77. Esta declaración está fundada en la regula fidei concedi-

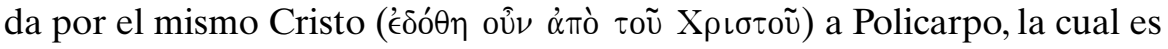
descrita como "la regla eclesiástica católica de la recta enseñanza

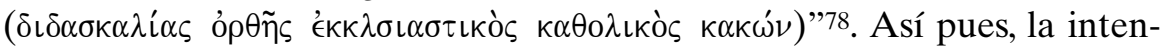
ción del autor de la Vida de Policarpo es subrayar contra la doctrina montanista que la Iglesia tiene el dominio exclusivo del Espíritu.

74 Cf. Eus., h. e. 5, 16, 10.

75 Gracias a Eusebio conocemos algo de esta actividad literaria y sus autores como Milciades, Apolinar de Hierápolis, Apolonio y el Anónimo Antimontanista, cf. Eus., h.e . 4, 27; 5, 16-19

76 Cf. M. Simonetti, Il vangelo e la storia, 80-81.

77 V. Polyc., 13, 3.

78 V. Polyc., 12, 2 
Significativa también es la estrategia antimontanista adoptada por el autor de la Vida de Policarpo al redefinir la profecía como visionaria y no como entusiasta, a través de la frecuente mención de visiones ${ }^{79}$ y la absoluta ausencia de cualquier profecía emitida a través de la posesión de un trance o de la suspensión de las funciones intelectivas que hacen que el supuesto profeta emita el mensaje en estado de inconciencia. Así pues, la profecía es emitida no cuando la persona se encuentra en un estado desordenado de su ánimo sino cuando el profeta en posesión de todas sus facultades, presumiblemente como un resultado de una visión, emite tal profecía ${ }^{80}$.

Por último, respecto a la Vida de Policarpo y los montanistas, el prólogo abrupto de la misma condena explícitamente la práctica pascual impropia de los herejes frigios que celebraban la Pascua fuera del tiempo de los ázimos, los cuales encontrando difícil calcular el 14 del mes lunar hebraico, en un ambiente helenístico en el que estaba en uso el calendario solar, al parecer se replegaron sobre el 14 del mes solar de Xanthicos que no coincidía con la antigua fecha pascual ${ }^{81}$.

De la mano con el desarrollo paulatino de la distinción entre una ortodoxia y grupos heréticos de variada denominación (marcionismo, gnosticismo, montanismo, etc.), se va desarrollando en la comunidad cristiana una reflexión cada vez mayor sobre la doctrina en torno a la persona de Cristo. Marción y sobretodo los gnósticos hacia la tercer decenio del siglo II habían asumido la cristología alta presente tanto en la tradición paulina como en la tradición juánica, reelaborando a partir de sus concepciones, el concepto de Cristo Logos cual entidad divina distinta del Dios sumo y a Él subordinada. Por otra parte, la doctrina del Cristo Logos también a mitad del siglo II comenzó a ser elaborada en ámbito católico, sobretodo en autores de proveniencia asiática depurándola de aspectos específicamente gnósticos y confesando a Cristo como Logos divino, ab aeterno inmanente en Dios como su palabra interna, sabiduría y pensamiento, generado (emanado, proferido) como entidad personal distinta de Él y a Él subordinada para proveer a la creación, al gobierno

${ }^{79}$ Cf. V. Polyc., 21, 2.

80 Una idea similar aparece en Epiph., haer. 48, 2-13, como ha hecho notar A. Stewart-Sykes entre otros. Para otras eventuales alusiones antimontanistas en la Vida de Policarpo, cf. A. Stewart-Sykes, The Life of Polycarp, 36-42.

81 Ps. Chrys., In s. Pascha, hom. 7, 9 (SCh 46, 119). Esta praxis montanista es atestiguada desde el siglo IV y resistió incluso hasta el siglo VI. Aunque nada hace pensar que esta praxis no se hubiese desarrollado en el punto más álgido de la historia del montanismo durante el siglo III, cf. R. Cantalamessa, La Pasqua della nostra salvezza, Città del Castello 2007, 119. 
y a la salvación del mundo y del hombre. Estos autores católicos afirmaban que para realizar la salvación, el Logos descendió del cielo y se encarnó en María en modo real, contra la convicción gnóstica de que el cuerpo asumido por el Logos era apariencia, o en cualquier caso, no material (docetismo) ${ }^{82}$.

Esta doctrina del Logos, desarrollada tanto por los católicos como por los gnósticos con su respectiva especificidad doctrinal, tuvo una reacción hacia finales del siglo II en el ámbito asiático con Noeto de Esmirna. Esta reacción, definida más tarde como monarquiana o patripasiana, buscaba salvaguardar la concepción monoteísta de Dios contra los exponentes de la doctrina del Logos. Así pues la doctrina de Noeto afirmaba que "Cristo mismo es el Padre y que el mismo Padre fue generado, sufrió y murió" 83 y si entonces se profesa "que Cristo es Dios, ciertamente él mismo es el Padre, porque Dios es uno sólo. Y Cristo ha padecido, él que es Dios: por tanto ha padecido el Padre, porque él era el Padre" 84.

Por su parte, Hipólito en su homilía Contra Noeto a escasos inicios del siglo III considera que la convicción de Noeto de gozar de una particular inspiración divina es producida por un espíritu malvado. Hipólito, cuyo origen es asiático ${ }^{85}$ pero no podemos asegurar que sea un esmirniota, después de una exposición introductoria de los hechos que sucedieron en Esmirna en torno a Noeto, presenta en la primera parte de su homilía, con tono hostil y con finalidad apologética en sentido antiherético, la doctrina de este personaje excomulgado por el colegio de presbíteros de Esmirna. Hipólito hace una secuencia de textos bíblicos adoptados por Noeto y posteriormente les interpreta en el sentido contrario según su propia postura que entiende como ortodoxa. Los textos citados son tanto del Antiguo como del Nuevo Testamento. Estos textos usados por Noeto para sostener su hilo doctrinal aseguraban que Dios es uno sólo (Ex. 3, 6; 20,$3 ;$ Is. 44, 6), que Dios había sido visto en la tierra, donde había conversado con los hombres (Bar. 3, 36-38; Is. 45, 14-15), que Cristo era Dios (Rom. 9, 5), y por ende, que el único Dios se debía identificar con Cristo. Finalmente, Noeto había hecho uso de Io. 10, 30 y Io. 14, 9-10 para confirmar la identificación de Cristo con Dios Padre. Para combatir la doctrina monarquiana de Noeto, Hipólito repropone la doctrina del Logos, pero

82 Cf. M. Simonetti, Contro Noeto, 44-45.

83 Hipp., $C N 1,2$.

${ }^{84}$ Hipp., $C N 2,3$.

85 La estrecha afinidad estilística que conecta $C N 18$ con las homilías cuartodecimanas nos asegura, entre otras cosas, la formación y proveniencia asiática del autor, cf. M. Simonetti, Contro Noeto, 70. 
debidamente adaptada a la doble exigencia de combatir las ideas de Noeto y al mismo tiempo tomar adecuada distancia de la doctrina gnóstica, por lo cual el Contra Noeto de Hipólito goza de interesante novedad en el modo de tratar esta temática ${ }^{86}$.

En la tradicional distinción de dos momentos en la relación entre Dios y su Logos, el primero inmanente-impersonal (pensamiento, sabiduría, potencia o voluntad), y el segundo entidad divina generada y personalmente distinta de Dios, Hipólito en modo original y con mucha fineza configura sistemáticamente el momento de la generación divina del Logos con su encarnación, presentada como manifestación al mundo de aquel que antes era invisible. Esta encarnación permite que el Hijo antes solo definido así a modo de prolepsis ahora ha llegado a ser Hijo perfecto $(\tau \in \in \in(\lambda \in\llcorner O \varsigma)$. Con esta acentuación del significado de la encarnación en la relación Dios Padre-Logos Hijo se acompaña la insistencia de Hipólito de revelar la realidad de la carne asumida por el Logos, capaz de sujetarse a todas las limitaciones de la humanidad. Así pues, esta asunción de la humanidad de parte del Logos y a la vez la unicidad del Hijo y de Logos, contraria a una multitud ( $\pi \lambda \eta \theta \dot{v} \nu)$ de dioses, son afirmadas por Hipólito con una clara impostación antignóstica ${ }^{87}$. Por otra parte, Hipólito hace una elaboración doctrinal contra Noeto más atenta a la doctrina del Logos que busca no dejar espacio a la acusación de diteísmo, aún especificando bien la subsistencia individual de Dios Padre y de su Logos

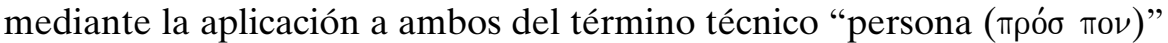

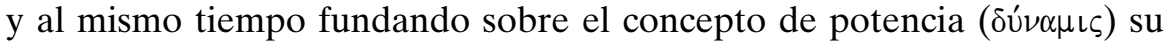
unidad, por la cual hablamos de ellos como un solo Dios.

Finalmente, la doctrina desarrollada por Hipólito en su Contra Noeto fue particularmente significativa en mencionar, aunque no siempre de modo armónico, al Espíritu Santo, pasando así del esquema binario (Padre-Hijo) al esquema ternario y ocasionalmente haciendo uso del tér-

86 Para un extenso comentario a la doctrina de Noeto y a la reacción por parte de Hipólito, cf. A. Orbe, Estudios sobre la teología cristiana primitiva, Madrid 1994, 71-95; y sobretodo, cf. M. Simonetti, Ippolito, Contro Noeto, Bologna 2000.

87 Cf. M. Simonetti, Contro Noeto, 47.

88 Tertuliano en África en su libro adversus Praxean también desarrolló de modo importante una doctrina sobre el Espíritu Santo. Autores posteriores como Novaciano en De Trinitate y el autor del Elenchos, por el contrario, no desarrollaron tanto así esta doctrina, muy probablemente debido a su reacción antimontanista, cf. M. Simonetti, Contro Noeto, 52 y 60. Por lo demás, la doctrina monarquiana de Noeto se difundió en Asia y especialmente en Roma con relativo éxito gracias a Epígono y Cleómenes en el tiempo de Ceferino y Calixto. El autor del Elenchos en su rivalidad con Calixto continuó la problemática en torno a la relación entre Dios y Cristo en el ámbito de la única divinidad dando origen a una polémica destinada a continuar por mucho tiempo. 
mino "trinidad ( $\tau \rho\llcorner\alpha$ $\varsigma)$ ". Esta mención del Espíritu Santo junto con el Padre y el Hijo usada desde hace tiempo por la lex orandi en la Iglesia de Esmirna y en todo el orbe cristiano, inspirada sobretodo por la fórmula de fe bautismal de $M t .28,19$, comenzó sólo gradualmente a hacerse paso también en el ámbito de la reflexión doctrinal (lex credendi). Como afirma M. Simonetti, puede ser que la primera hora del movimiento montanista, que hemos referido más arriba, haya impulsado un poco en autores como Hipólito en Asia la reflexión doctrinal sobre el Espíritu Santo. Sin embargo, justamente porque cada vez más la actividad carismática fue tan valorizada por los seguidores de Montano y estos fueron percibidos cada vez más como una secta, es que la reacción antimontanista paralizó por más de un siglo el desarrollo de la reflexión sobre el Espíritu Santo ${ }^{88}$.

\section{Conclusión}

Con la consolidación paulatina de la lex orandi de la comunidad se va haciendo mayor distinción entre la ortodoxia y los grupos heréticos. En la primera mitad del siglo II la comunidad es amenazada por cierto grupo de cristianos docetas y además una parte de la comunidad parece no reconocer la figura del obispo ni el ordenamiento jerárquico a tres niveles. La presencia de cristianos judaizantes que puedan estar involucrados en estas situaciones es probable. Por otra parte, es difícil individuar la presencia o alusión a los gnósticos en los escritos de Ignacio o

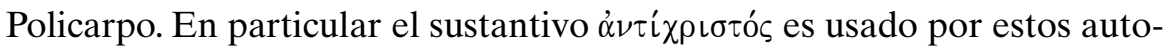
res para definir al falso maestro y falso hermano que va en contra de la tradición recibida.

En la segunda parte del siglo II y durante el siglo III aparece clara la paulatina individuación y controversia con el marcionismo y, sobretodo, con el movimiento de la Nueva Profecía en los escritos de la comunidad, particularmente en el Martirio de Pionio y en la Vida de Policarpo. Finalmente, es claro el rechazo explícito de la comunidad de Esmirna a la doctrina patripasiana de Noeto que identifica al Padre con el mismo Cristo y por tanto como aquel que ha padecido en la cruz.

A lo largo de este artículo, hemos visto cómo los diversos modelos de símbolos de fe que se encuentran en el Nuevo Testamento también están presentes en la documentación de la Iglesia de Esmirna y son usados tanto en contextos catequéticos, bautismales y litúrgicos como también en contextos de persecución y controversia frente a paganos, judíos 
y herejes. El adverbio $\dot{\alpha} \lambda \eta \theta \tilde{\omega} \varsigma$, presente en varias confesiones de fe, señala el cuño antidoceta de estas, especialmente en los escritos de Ignacio y Policarpo. De considerable interés por la riqueza doctrinal y la armonía del texto es la oración que el autor del Martirio de Policarpo coloca en labios del obispo de Esmirna antes de su muerte.

Es particularmente importante la profesión de fe de los presbíteros de Esmirna en que se proclama la unicidad de Dios frente a las ideas gnósticas y marcionitas, a la vez que se hace la distinción entre Dios y Cristo frente a la coyuntura suscitada por Noeto y la confesión de la verdadera pasión, muerte y resurrección del Señor frente al docetismo. Este texto, aunque breve, es un punto de llegada seguro en el desarrollo doctrinal de la Iglesia de Esmirna, precisando de manera oficial y vinculante los conceptos fundamentales contenidos en la regla de fe. Esta confesión de fe de carácter normativo además pudo ser un símbolo de fe bautismal usado por la comunidad a finales del siglo II e inicios del siglo III. 